\title{
Lineamientos de bioseguridad utilizados en las clínicas dentales periféricas de la Universidad de Monterrey durante la pandemia de COVID-19.
}

\author{
Biosafety guidelines used in the peripheral dental clinics of the \\ University of Monterrey during the COVID-19 pandemic.
}

\author{
Selenia J Medina Arizpe, * Alfredo Salinas Noyola ${ }^{\ddagger}$
}

\section{RESUMEN}

El presente artículo tiene como propósito informar sobre los lineamientos que las clínicas dentales periféricas de la Escuela de Odontología de la Universidad de Monterrey (UDEM) han tomado frente a la pandemia actual COVID-19, emergente en la ciudad de Wuhan, China el pasado diciembre 2019. Dichas medidas surgen con el objetivo de cumplir con estándares de bioseguridad que eviten el contagio y/o la contaminación cruzada entre pacientes, profesionales y personal de las clínicas dentales de la UDEM; Clínica de Prevención Dental (CPD) y Clínica de Atención Dental Avanzada (CADA), las cuales permanecerán activas frente a situaciones que requieran atención de urgencia y postergando los tratamientos dentales de rutina. Los protocolos para brindar una atención de urgencia conllevan determinados pasos a seguir desde que el paciente ingresa a la clínica; iniciando con la revisión del expediente electrónico médico por medio de la plataforma Atlas.xp, seguido del llenado de un cuestionario enfocado al riesgo de la enfermedad y culminando con la toma de temperatura con termómetro infrarrojo. Los pacientes que no presenten síntomas y muestren una temperatura inferior a los $37.3^{\circ} \mathrm{C}$ podrán ser atendidos bajo la aplicación de todas las medidas de bioseguridad establecidas (medidas de protección personal, de equipo y paciente, mantenimiento de dos metros de distancia en sala de espera, cumplimiento de tiempos de trabajo parciales, uso de lámparas purificadoras de aire distribuidas en las salas operatorias de las clínicas, trabajo asistido o a cuatro manos con la implementación de aislamiento absoluto en el paciente y con la utilización mínima de la pieza de alta velocidad). Asimismo, se menciona que posterior a cada tratamiento y en la culminación de la jornada laboral, todas las áreas y superficies deberán ser sanitizadas con sustancias desinfectantes

\section{ABSTRACT}

The purpose of this article is to inform about the guidelines that the peripheral dental clinics of the School of Dentistry of the University of Monterrey (UDEM) have taken in the face of the current pandemic COVID-19, emerging in the city of Wuhan, China in the past December 2019. These measures arise with the aim of fulfilling biosafety standards that avoid contagion and/or cross contamination between patients, professionals and staff of UDEM dental clinics; Clínica de Prevención Dental (CPD) and Clínica de Atención Dental Avanzada (CADA), which will remain active in situations that require urgent care and postponing routine dental treatments. The protocols to provide urgent care include certain steps to follow from the moment the patient enters the clinic; starting with the review of the electronic medical record through the Atlas.xp platform, followed by the completion of a questionnaire focused on the risk of the disease and culminating with temperature measurement with an infrared thermometer. Patients who do not present symptoms and show a temperature below $37.3^{\circ} \mathrm{C}$ may be treated under the application of all established biosecurity measures (personal, equipment and patient protection measures, maintenance of two meters in the waiting room, compliance with partial work times, use of air purifying lamps distributed in the operating rooms of the clinics, assisted or four-hand work with the implementation of absolute isolation in the patient and with the minimum use of the high-speed handpiece). It is also mentioned that after each treatment and at the end of the working day, all areas and surfaces must be sanitized with specific disinfecting substances. For their part, the personnel must wear conventional clothing when leaving the facilities and have carried out adequate hand washing to avoid transmission of the virus as much as possible.

\footnotetext{
* Catedrático y coordinador clínico.

₹ Director de la Escuela de Odontología.
}

Universidad de Monterrey. 
específicas. Por su parte, el personal deberá portar ropa convencional al salir de las instalaciones y haber realizado un adecuado lavado de manos para evitar al máximo posible la transmisión del virus.

Palabras clave: SARS-CoV-2, bioseguridad, COVID-19, odontología, equipo de protección personal.

\section{INTRODUCCIÓN}

E n enero del presente año, la Organización Mundial de la Salud (OMS) anunció una emergencia de salud pública mundial, el nuevo coronavirus COVID-19, que ocasiona el síndrome respiratorio agudo grave (SARS), que inició en diciembre de 2019 en la ciudad de Wuhan, China. Lo anterior se ha convertido en un problema no sólo de ciudades aledañas a China, sino en una crisis global. ${ }^{1,2}$ Debido a las características del trabajo en el área odontológica, hay un riesgo mayor de tener infecciones cruzadas entre el odontólogo y los pacientes. ${ }^{1,2}$ Las ciudades afectadas por el COVID-19 deberán tener estándares altos de bioseguridad para el control de infecciones cruzadas y control de aerosoles en el consultorio dental. ${ }^{1,2} \mathrm{El}$ presente artículo describe los lineamientos de bioseguridad implementados en las clínicas periféricas de la Escuela de Odontología de la Universidad de Monterrey.

\section{ETIOLOGÍA VÍRICA DEL COVID-19}

El SARS-CoV-2 es zoonótico, proviene del murciélago de la región de China Rhinolophus sinicus, siendo el pangolín el principal intermediario. ${ }^{1,2}$ La carne del pangolín es exótica y muy utilizada en la región de Asia, y actualmente se encuentra en peligro de extinción debido al uso de sus escamas para tratar el asma y alguna otra enfermedad respiratoria como parte de la medicina tradicional oriental. ${ }^{3}$

\section{RUTAS DE TRANSMISIÓN}

La ruta de contaminación inicia de un solo contacto entre animal-humano, seguido de humano a humano por medio de las gotas derivadas del tracto respiratorio o flugge, además de la transmisión orofecal. También se conoce la transmisión vertical. ${ }^{1}$

\section{FUENTES DE TRANSMISIÓN}

Además de los pacientes sintomáticos con COVID-19, que son la fuente principal de contagios, recientemente se
Keywords: SARS-CoV-2, biosecurity, COVID-19, dentistry, equipment personal protective. ha sugerido que los pacientes asintomáticos y pacientes en periodo de incubación son portadores del SARS-CoV-2. Esto ha ocasionado la dificultad para identificar a los pacientes infectados, resultando en acumulación de los mismos en las comunidades infectadas. ${ }^{1}$

\section{PERIODO DE INCUBACIÓN}

El periodo de incubación del COVID-19 se ha estimado entre cinco a seis días en promedio; sin embargo, existe evidencia de que puede durar hasta 14 días en incubación después de la primera exposición de la persona hacia el virus. ${ }^{1,2}$

\section{POBLACIÓN DE MAYOR RIESGO DE INFECCIÓN}

Todas las edades poblacionales tienen riesgo a contagio, sin embargo, los médicos y profesionales de la salud tienen mayor potencial de ser contagiados por la infección del SARS-CoV-2 ya que el virus puede permanecer en superficies por algunas horas hasta días, dependiendo el tipo de superficie, temperatura y humedad. ${ }^{1}$

\section{MANIFESTACIONES CLÍNICAS}

De acuerdo con estudios recientes, la mayoría de los casos COVID-19 son relativamente con sintomatología moderada y según la Comisión Nacional de Salud de China, la proporción de casos severos entre pacientes con COVID-19 fue alrededor de 15 a 25\%. ${ }^{1}$

La mayoría de los pacientes experimentaron: fiebre, tos seca, falta de aliento, fatiga y algún otro síntoma atípico como dolor muscular, confusión, dolor de cabeza, dolor de garganta, diarrea y vómito. ${ }^{1}$

\section{DIAGNÓSTICO Y PLAN DE TRATAMIENTO}

El diagnóstico de COVID-19 se puede basar en información epidemiológica, signos y síntomas clínicos, 
exámenes de laboratorio prueba de CPR positiva, tomografía computarizada de tórax. Cabe mencionar que una sola prueba de CPR negativa no excluye la infección. Actualmente no existe ningún tratamiento específico para esta enfermedad, sólo el manejo específico de los síntomas. ${ }^{1}$

\section{PROTOCOLO DE BIOSEGURIDAD EN LAS CLÍNICAS UDEM BAJO LA PANDEMIA COVID-19}

El SARS-CoV-2, es más estable en plástico; acero inoxidable que en el cobre, y cartón; el virus es detectable hasta 72 horas después. La transmisión del virus es plausible a través de los aerosoles en las superficies que emiten las personas infectadas, o incluso hasta días después dependiendo del porcentaje de humedad del cuarto. ${ }^{4}$ Debido a lo anterior, a la emergencia sanitaria mundial y conforme a las guías de atención bucodental en clínicas de segundo nivel de atención, se establecen las siguientes recomendaciones:

Identificar los procedimientos de urgencia y emergencia en la práctica odontológica.

Se consideran emergencias odontológicas:

- Sangrado activo o descontrolado.

- Celulitis o infección bacteriana difusa de los tejidos blandos con edema extraoral que comprometa las vías respiratorias del paciente.

- Traumatismos que involucren huesos faciales y las vías aéreas respiratorias.

Estas emergencias son referidas a nivel hospitalario para su manejo integral.

\section{UDEM}

Fecha: $\_l-1$

\section{Cuestionario COVID-19}

Nombre del paciente:

Atendido por:

1. ¿Presenta o ha presentado fiebre en los últimos 14 días?

2. ¿Ha presentado problemas respiratorios, como tos o dificultad para respirar en los últimos 14 días?

3. ¿Durante los últimos 14 días ha viajado a Asia, Europa o países endémicos, o ha visitado a alguna persona que ha sido documentada con transmisión de COVID-19?

4. ¿Ha estado en contacto con algún paciente confirmado con COVID-19 en los últimos 14 días?

5. ¿ ¿Ha estado en contacto con personas que vienen de Asia, Europa o países endémicos y que han presentado problemas respiratorios en los últimos 14 días?

6. ¿Ha tenido contacto con personas que han experimentado problemas respiratorios y fiebre durante los últimos 14 días?

7. ¿Ha participado recientemente en conglomeraciones, reuniones o ha tenido contacto cercano con personas inconscientes de la situación?

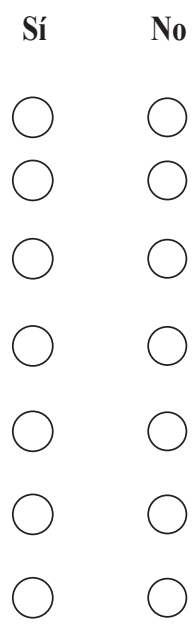

Temperatura: ${ }^{\circ} \mathrm{C}$

Figura 1: Cuestionario para paciente. 


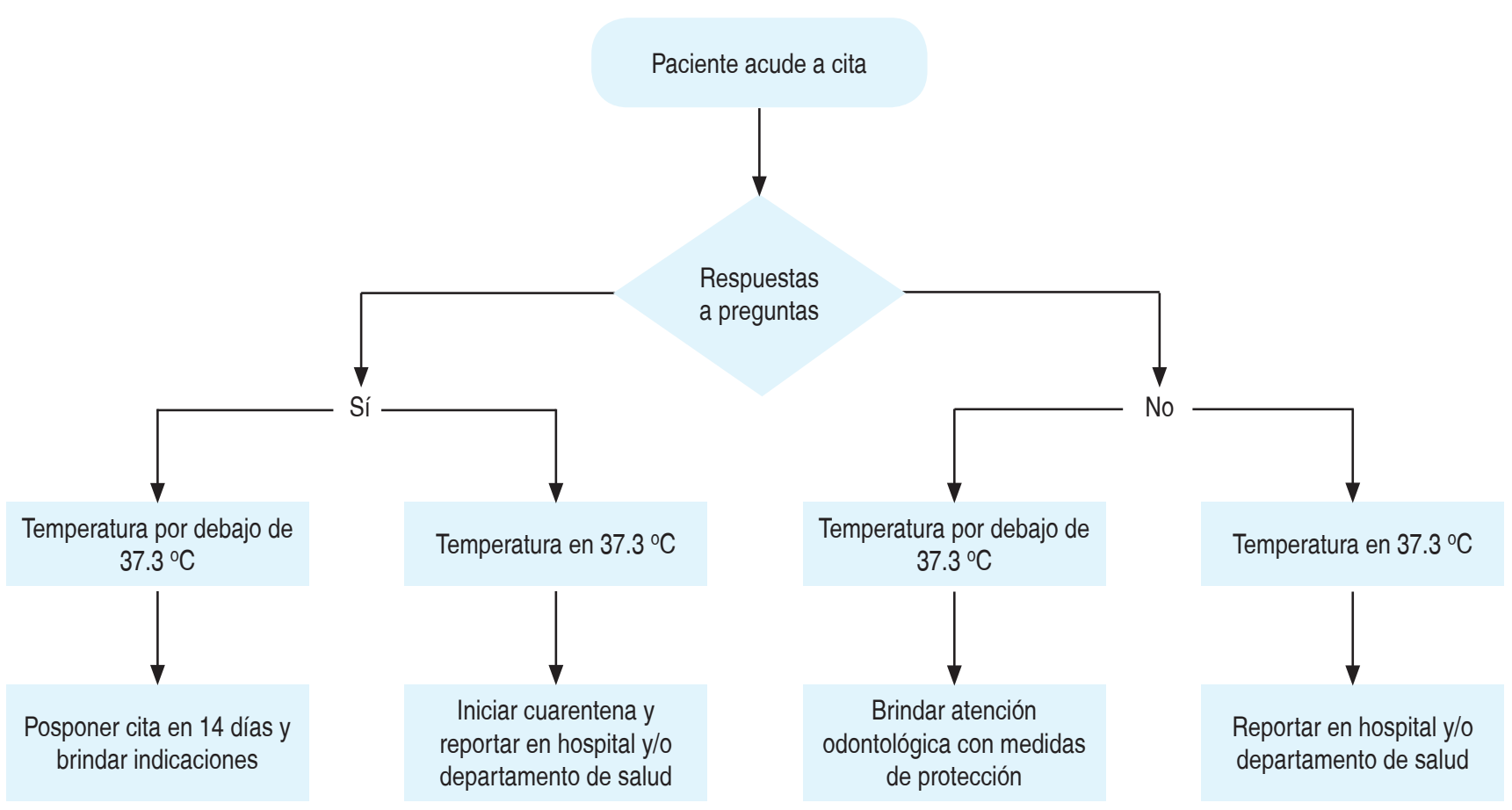

Si algún paciente presenta síntomas o es sospechoso referir a su IMSS o Centro de Salud (SSA)

Figura 2: Diagrama de flujo para aceptación e ingreso de paciente.

Se consideran urgencias odontológicas:

- Dolor dental por pulpitis reversible o irreversible.

- Pericoronitis.

- Osteítis postoperatoria.

- Alveolitis.

- Absceso o infección localizada derivado de un órgano dental.

- Traumatismo dental con avulsión y/o luxación.

- Cementación de restauración temporal provocando irritación gingival.

- Fractura de una restauración que provoque dolor intenso.

Cabe señalar que cualquier tratamiento de atención odontológico no mencionado entra en una categoría de tratamiento dental de rutina y queda postergado. ${ }^{2}$

\section{CAPACITACIÓN AL PERSONAL ODONTOLÓGICO}

Por parte de la Universidad de Monterrey y de la Escuela de Odontología, se implementaron cursos y clases en línea para capacitar al personal administrativo y profesores de la escuela para entender la patogenia del virus y las medidas de bioseguridad para evitar contagios y contaminación cruzada. ${ }^{1,2,5,6}$

\section{TRIAGE ODONTOLÓGICO}

Se establece el triage odontológico por medio de la telemedicina con la revisión del portal Atlas.xp, herramienta de expediente electrónico donde se revisan las notas de evolución del paciente, evaluando así atención de las posibles urgencias o emergencias dentales de nuestros pacientes. Asimismo, se realiza el llenado de una encuesta electrónica (Figura 1) enfocada al riesgo de la enfermedad con las siguientes preguntas:

Se utiliza un diagrama de flujo para determinar si el paciente puede ser intervenido o no (Figura 2). ${ }^{2}$ Una vez evaluado, la urgencia y que el paciente se encuentre en clínica, se realiza la toma de temperatura corporal de cada paciente por medio de un termómetro infrarrojo, llevando así un registro de cada uno de ellos. La atención sólo se brindará a una persona por hora, para evitar la 
contaminación cruzada por flugge. Adicional a esto, existe una delimitación de $60 \mathrm{~cm}$ en la recepción (Figura 3).

Antes de ingresar a la clínica, se le solicita al paciente pasar a lavarse las manos por 20 segundos con la técnica ejemplificada en la zona de cepilleros y se le ofrece gel antibacterial alcoholizado al $70 \% .^{5}$

\section{SALA DE ESPERA}

Se solicita al paciente que acuda sin acompañante. Solamente puede ir acompañado en caso de que requiera asistencia o sea menor de edad. Son registrados los datos del paciente y del acompañante, así como la toma de temperatura de ambos; si el paciente o su acompañante presenta una temperatura mayor a $37.3^{\circ} \mathrm{C}$, la consulta será postergada para después y se canalizará al paciente a alguna institución de salud para su seguimiento médico. ${ }^{2,4,5}$

Se distribuyeron las sillas estratégicamente a dos metros de distancia entre ellas para mantener la distancia adecuada para evitar contagio por flugge. Se adecua una área para la colocación de cubrecalzado, gorro desechable y cubrebocas para el paciente (Figura 4).

\section{EQUIPO DE TRABAJO}

Para el personal administrativo y operativo de la clínica se establecieron tiempos de trabajo parciales: cuatro horas por jornada para las asistentes, cuatro horas para los coordinadores de clínica y tres jornadas de cinco horas para el docente bajo el esquema de contratación de planta. Esto con el fin de evitar aglomeraciones por

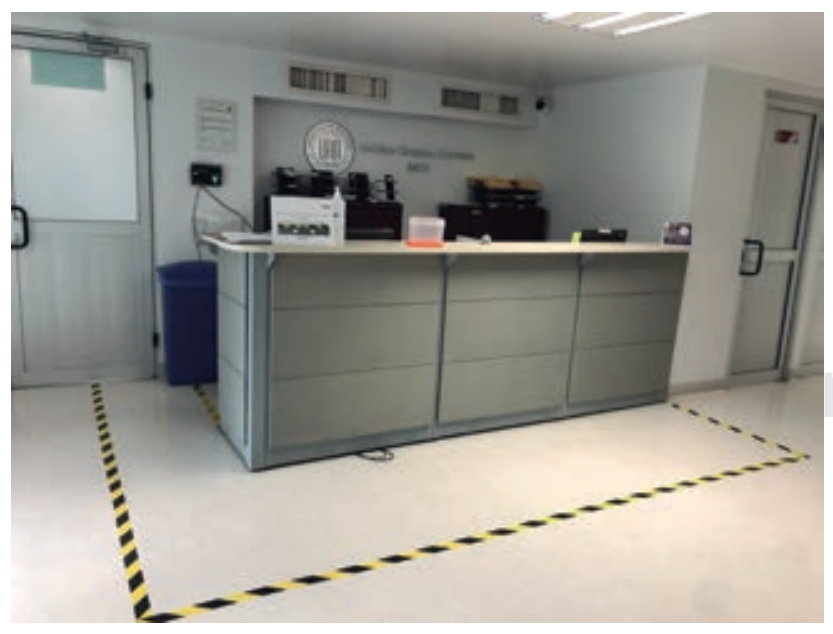

Figura 3: Delimitación de recepción de pacientes.

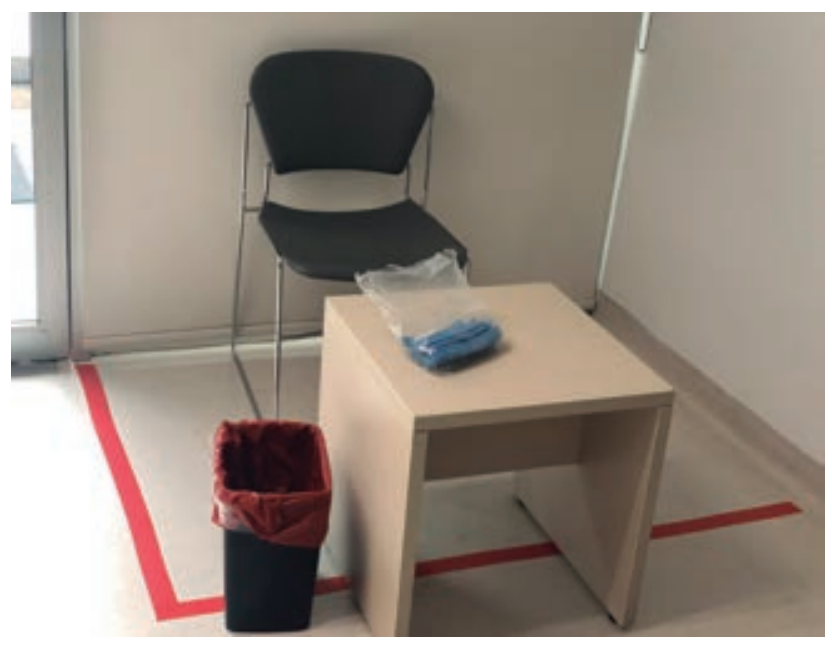

Figura 4: Espacio designado para colocación de barreras para calzado y aplicación de gel antibacterial.

parte del personal de salud y que de igual manera antes de entrar a clínica es registrada la temperatura corporal de cada profesional. Los pasantes de la Secretaría de Salud acuden solamente una jornada de seis horas a la semana.

\section{EQUIPO RADIOLÓGICO}

Para evitar la exposición continua al exudado nasofaríngeo de los pacientes en la toma de radiografía intraoral, se sugiere la toma de radiografías extraorales o cone-beam para solucionar las emergencias y urgencias dentales en la medida posible. El equipo de ortopantomografía cuenta con la opción de toma de aletas mordibles, así como la manipulación de la imagen para obtener mayor calidad de las mismas.

\section{ATENCIÓN ODONTOLÓGICA Y EQUIPO DE PROTECCIÓN PERSONAL}

Se le proporciona a todo el personal odontológico las medidas de protección personal (EPP), las cuales incluyen:

- Gorro desechable.

- Bata desechable con puño.

- Cubrebocas N95.

- Guantes de nitrilo.

- Pijama quirúrgica especial antiderrapante.

- Careta protectora.

- Lentes de protección o lentes de aumento en su defecto.

- Cubrezapatos. 


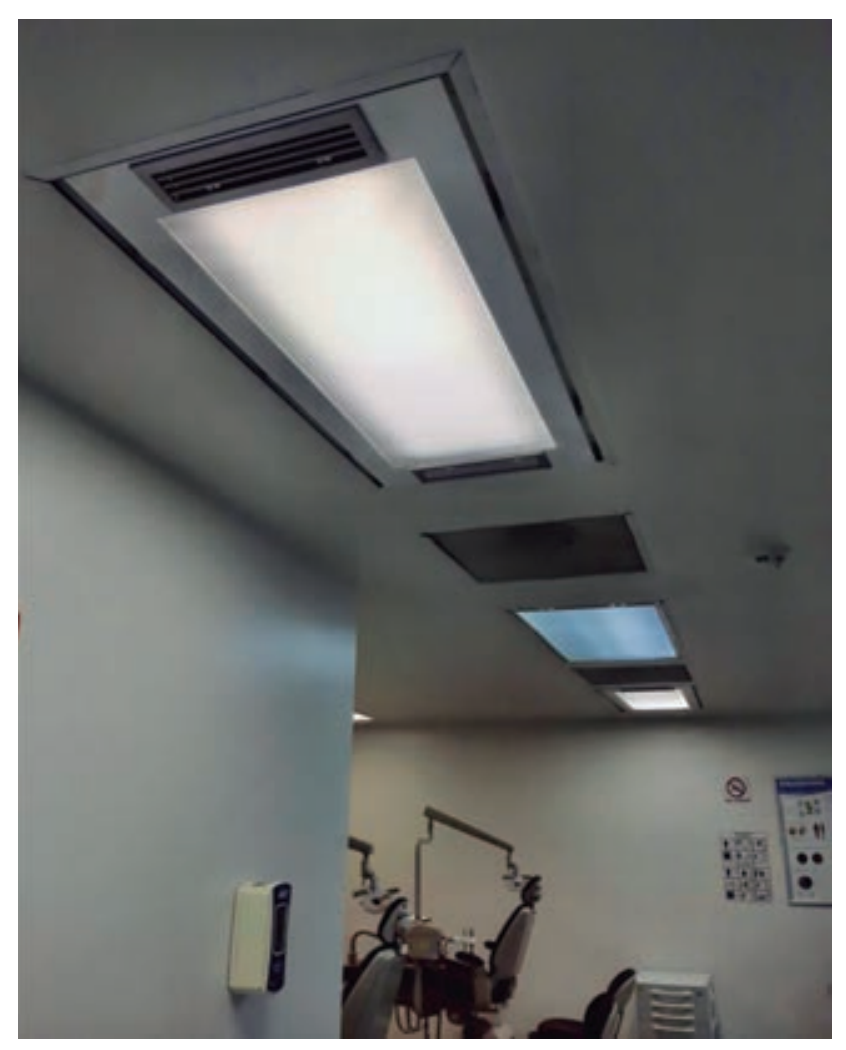

Figura 5: Colocación de lámparas de purificación de aire y disminución de bacterias.

A nivel de infraestructura, se colocaron de manera estratégica lámparas de purificación continua y rápida de aire con luz ultravioleta tipo $\mathrm{C}$, distribuidas de la siguiente manera: cuatro para la Clínica de Prevención Dental y siete para la Clínica de Atención Dental Avanzada (edificio de dos plantas). Es un sistema que filtra el aire con la finalidad de disminuir el asentamiento de hongos y bacterias, mejorando así la calidad del aire de la zona operatoria de ambas clínicas (Figura 5).

El sillón dental debe seguir un protocolo de sanitización con una solución que contiene etanol, cloruro didecil-dimetil amonio y agua. Una vez concluido el paso anterior, se procede a la colocación de protecciones de plástico para eyector, lámpara, mango del carro de instrumental, jeringa triple, bracket de la unidad (Figura 6); el instrumental mantiene su protocolo de lavado y esterilizado, que va desde la colocación de los instrumentos en el ultrasonido y posteriormente en bolsas de esterilización. Se recomienda el uso de puntas desechables para la jeringa triple, aun cuando se esterilice la convencional.
Una vez que el paciente ingrese a la clínica para su atención odontológica, se solicita realizar un enjuague con peróxido de hidrógeno al $5 \%$ durante 30 segundos sin escupir, sólo dejando caer el líquido al término del tiempo. 5,6 Se evitará a toda costa cualquier tratamiento que genere aerosol de una pieza rotatoria, siendo los tratamientos con técnica TRA o de mínima invasión utilizando cucharilla de dentina para retirar tejido infectado, los ideales en esta pandemia. ${ }^{5}$ En caso de utilizar pieza de mano de alta velocidad se implementa el aislamiento absoluto desde la apertura de la cavidad, si es que así lo requiere el paciente, reduciendo esto en un $70 \%$ la cantidad de aerosoles generada. ${ }^{2,5}$ Se trabaja a cuatro manos con un asistente que porte la misma cantidad de barreras. ${ }^{2,5}$

Al final de la atención odontológica se realizará la asepsia de las superficies y áreas comunes con hipoclorito de sodio $\geq 0.21 \% ;{ }^{5}$ las líneas de succión son tratadas con una fórmula multienzimas, que elimina detritos, saliva y residuos de sangre, evitando un acúmulo de sólidos. Estos cuidados son llevados a cabo por el personal de intendencia, quienes a su vez portarán la misma cantidad de barreras de protección, sólo que con un cubrebocas de tres capas. ${ }^{5} \mathrm{Al}$ finalizar la jornada laboral se le pedirá a todo el personal, colocarse su ropa convencional para no contaminar los medios de transporte, así como el lavado de manos y la toma de temperatura corporal.

\section{AGRADECIMIENTO}

Agradecimiento especial a todo el personal de salud por su gran labor ante esta pandemia.

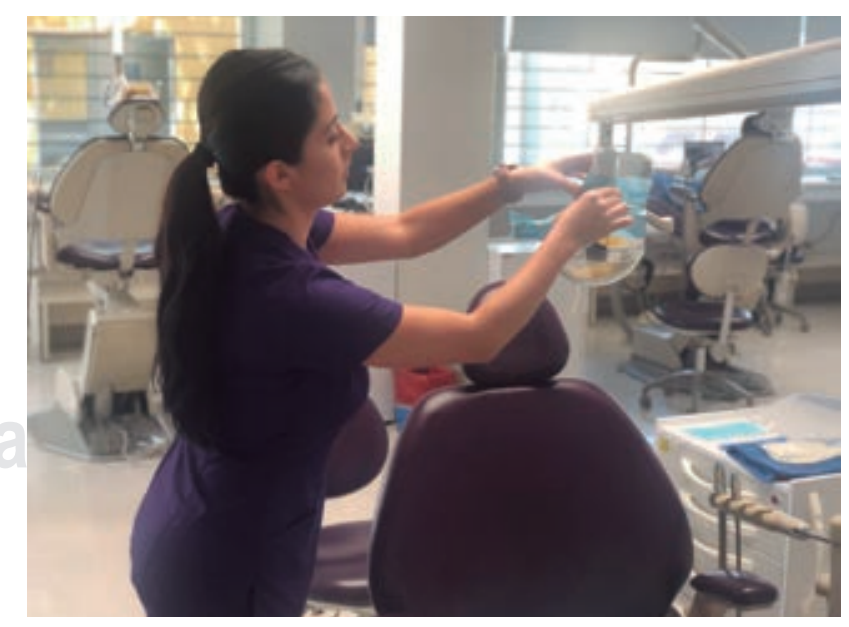

Figura 6: Sanitización de superficies del sillón dental y colocación de barreras. 


\section{BIBLIOGRAFÍA}

1. Meng L, Hua F, Bian Z. Coronavirus disease 2019 (COVID-19) Emerging and Future Challenges for Dental and Oral Medicine. J Dent Res. 2020; 99 (5): 481-487.

2. Peng X, Xu X, Li Y, Cheng L, Zhou X, Ren B. Transmission routes of 2019-n CoV and controls in dental practice. Int J Oral Sci. 2020; 1-7.

3. Lam TT, Shum MH, Zhu HC, Tong YG, Ni XB, Liao YS et al. Identifying SARS-CoV-2 related coronaviruses in Malayan pangolins. Nature. 2020; 1 -3.

4. Aerosol and Surface Stability of SARS-CoV2 as Compared with SARS-CoV1. N Engl J Med. 2020; 382: 1564-1567.

5. Asociación Latinoamericana de Odontopediatría. 2020. Ruta de atención para procedimientos de Odontología Pediátrica durante la etapa de confinamiento o cuarentena por la pandemia del COVID-19.

6. American Academy of Pediatric Dentistry. 2020 COVID-19 Update.

\section{LECTURAS RECOMENDADAS}

- Pediatric Dentistry During and After COVID-19. Pediatr Dent. 2020; 42 (2): 87-90.

- https://www.cdc.gov/vaccines/adults/rec-vac/hcw.html.

- https://www.cdc.gov/oralhealth/infectioncontrol/statement-COVID.html.

- https://www.ada.org/en/publications/ada-news/2020-archive/ march/ada-recommending-dentists-postpone-elective-procedures.

- https://www.who.int/es/emergencies/diseases/novelcoronavirus-2019.

\section{Correspondencia:}

\section{Dr. Alfredo Salinas Noyola}

Universidad de Monterrey

E-mail: alfredo.salinas@udem.edu 